\title{
I got rhythm, I got music
}

Last year, Simiak Baharloo and colleagues showed that absolute pitch - the ability to identify precisely and rapidly the pitch of a note - has a substantial genetic component (see Highlights, October 2000). Now, Dennis Drayna and colleagues focus on relative pitch (RP) - the ability to perceive pitch alterations — and find that it also has a surprisingly large genetic component.

To investigate the relative contributions of genetics and the environment to RP perception, Drayna et al. used a twin study and a modernized distorted tunes test (DTT), which aims to minimize the effects of cultural bias and musical training. They tested 136 monozygotic (MZ) and 148 dizygotic (DZ) Caucasian female twins for their ability to spot false notes in popular tunes. Statistical analysis of the results revealed a significant difference in the DTT scores between $\mathrm{MZ}$ and $\mathrm{DZ}$ twins, which indicates a strong genetic component to RP perception.

\section{FUNCTIONAL GENOMICS}

\section{Networking skills}

A substantial proportion of the genes in any genome has no known function. In yeast, this 'function gap' is being tackled using several genome-wide approaches, and Ito et al. have now published the results of a systematic two-hybrid screen, which

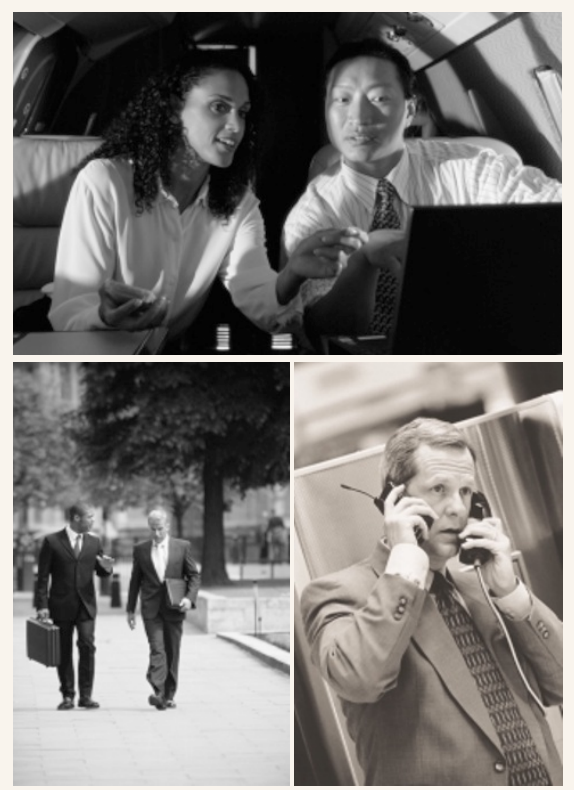

Additionally, Drayna et al. showed that RP perception is largely independent of age and peripheral hearing - the ability to hear sounds. This last finding indicates that RP and peripheral hearing might involve separate parts of the auditory system.

The correlation of DTT scores for the MZ data set was calculated at 0.67 compared with 0.44 for the DZ data set. Sophisticated statistical data modelling indicated a strong influence of additive genetic factors and a small environmental contribution, for example from musical training. The same models establish heritability, which is the proportion of variance attributable to genetic differences, at $0.71-0.80$ - a very high score for a complex genetic trait. But the authors point out that the results will need to be replicated in other populations.

By showing that RP perception might be partly 'hardwired', this study has struck a chord among cognitive scientists who are studying

provides a large collection of new data on protein-protein interactions. A similar screen was published by Uetz et al. last year, and a comparison of the two data sets allows Ito et al. to conclude that this is a valuable approach for identifying novel gene functions, but that the individual studies are far from complete.

The two-hydrid screen involves two plasmid constructs that each express a fusion protein - between test protein $X$ and the yeast Gal4 DNA-binding domain, and between test protein $\mathrm{Y}$ and the Gal4 activation domain. A reporter gene that is regulated by Gal4 will be activated if proteins $\mathrm{X}$ and $\mathrm{Y}$ interact. This provides the basis for a simple genetic screen for interactions between any two gene products in the yeast genome. Ito $e t$ al. pooled sets of constructs, such that each pool expressed either 96 DNA-binding fusion proteins or 96 activation-domain fusion proteins. By using all pairwise combinations of the pools, they screened the entire set of $\sim 6,000$ yeast open reading frames (ORFs) for protein-protein interactions.

Two-hybrid screens are subject to falsepositive errors, so to ensure that the results of the screens reflect real protein interactions, stringent criteria are applied to the data. For example, Ito et al. scored an interaction as positive only if it was identified on at least three independent

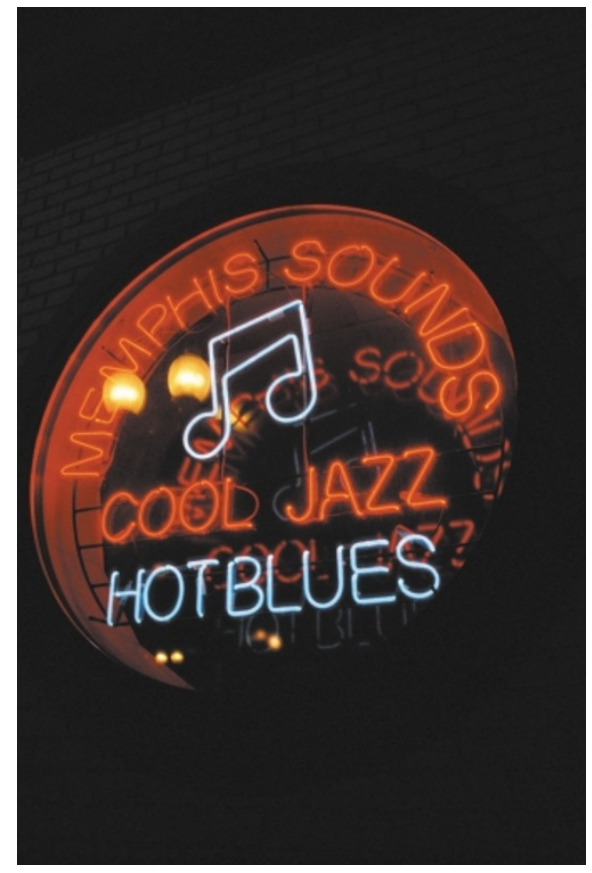

communication disorders, and who noticed a long time ago that musicality and language ability go together. They might now be encouraged to look for the genetic basis of such disorders.

(4) References and links

Magdalena Skipper

ORIGINAL RESEARCH PAPER Drayna, D. et al. Genetic

correlates of musical pitch recognition in humans. Science $\mathbf{2 9 1}$ 1969-1972 (2001)

FURTHER READING Holder, C. Study suggests pitch perception is inherited. Science 291, 1879 (2001) WEB SITE Dennis Drayna's lab | Distorted tunes test WEB SITE Dennis Drayna's lab Distorted tunes test
ENCYCLOPEDIA OF LIFE SCIENCES Quantitative genetics

occasions. Overall, they found such evidence for $\sim 800$ interactions and, when compared to the $\sim 700$ interactions identified by Uetz et al., there were only 141 interactions in common between the two screens. The clear implication is that neither study has identified all yeast protein interactions, perhaps because of the different strategies used to reduce the false-positive error rate.

There is also little doubt that these studies provide valuable functional information about genes of unknown function, potential protein complexes and novel links between different cellular functions. Ultimately, this information will be summarized in a comprehensive protein interaction network for the cell. The relatively small overlap between the first two systematic protein-interaction studies and the new functional insights that these data provide should encourage similar efforts using variations on this and other proteomic techniques.

Mark Patterson

6) References and links ORIGINAL RESEARCH PAPER Ito, T. et al. A comprehensive two-hybrid analysis to explore the yeast protein interactome. Proc. Natl Acad. Sci. USA 98, 4569-4574 (2001)

FURTHER READING Uetz, P. et al. A comprehensive analysis of the protein-protein interactions in Saccharomyces cerevisiae. Nature 403, 623-627 (2000) WEB SITE Takashi Ito's lab | Database of interacting proteins 